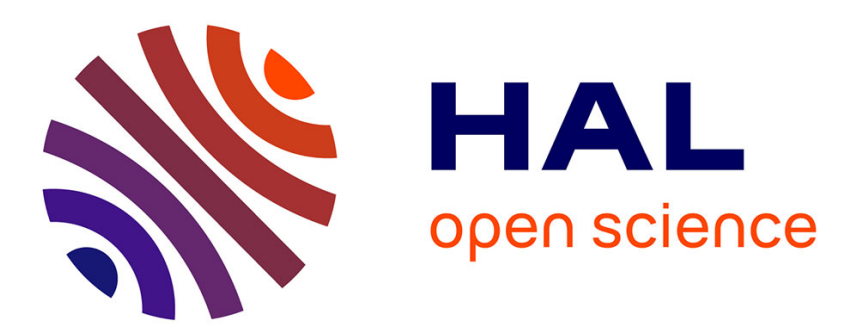

\title{
Continuous nonlinear eigenvalue solver with applications to the design of electro/magnetorheological sandwich structures
}

V Ammovilli, M Bilasse, I. Charpentier

\section{- To cite this version:}

V Ammovilli, M Bilasse, I. Charpentier. Continuous nonlinear eigenvalue solver with applications to the design of electro/magnetorheological sandwich structures. Smart Materials and Structures, 2019. hal-02333183

\section{HAL Id: hal-02333183 \\ https://hal.science/hal-02333183}

Submitted on 25 Oct 2019

HAL is a multi-disciplinary open access archive for the deposit and dissemination of scientific research documents, whether they are published or not. The documents may come from teaching and research institutions in France or abroad, or from public or private research centers.
L'archive ouverte pluridisciplinaire HAL, est destinée au dépôt et à la diffusion de documents scientifiques de niveau recherche, publiés ou non, émanant des établissements d'enseignement et de recherche français ou étrangers, des laboratoires publics ou privés. 


\title{
Continuous nonlinear eigenvalue solver with applications to the design of electro/magnetorheological sandwich structures
}

\author{
V Ammovilli ${ }^{1}$, M Bilasse $^{1,2}$ and I Charpentier ${ }^{1}$ \\ ${ }^{1}$ Icube UMR 7357, Université de Strasbourg \& CNRS, 4 rue Boussingault, \\ 67000 Strasbourg, France \\ 2 ECAM Strasbourg Europe, 2 rue de Madrid, 67300 Schiltigheim, France \\ E-mail: vammovilli@unistra.fr
}

\begin{abstract}
Smart sandwich structures comprising an electro- or a magnetorheological material have the potential to attenuate vibration over a wide range of frequencies. The analysis of their vibration behaviour with respect to the continuous variation of the field intensity is thus a major challenge for research and industry to maximize damping treatments. The numerical higher order homotopy method we propose models the effects of a continuous variation of the field intensity on resonant frequencies and loss factors by means of Taylor expansions. Comparisons between our continuous approach and the classical incremental method are proposed for state of the art sandwich beams and plate structures comprising ER/MR fluids to highlight the benefits of our continuous methods in terms of maximal damping determination.
\end{abstract}

Keywords: Smart sandwich structures, nonlinear free vibration, homotopy method, Taylor series, automatic differentiation.

Submitted to: Smart Mater. Struct. 


\section{Introduction}

Sandwich structures are defined as multilayered composite structures, optimized for desired/anticipated lifetime loading conditions occurring in engineering applications such as automotive, railway, submarine, and aerospace. The analysis of their vibration behaviour is a major challenge for industry and research from experimental and numerical points of view.

The benefits of sandwich structures involving viscoelastic (VE), electrorheological (ER) or magnetorheological (MR) damping treatments are demonstrated for vibration attenuation or suppression [1, 2]. Sandwich structures with their fixed VE material parameters usually yield limited vibration attenuation within a narrow frequency band. Recent advances on VE sandwich structures are reviewed in 3 .

The rheological properties (viscosity and shear modulus) of ER/MR fluids or elastomers are functions of the applied external field respectively. When such a material is embedded between two elastic layers [4, 5], the stiffness and damping properties of the sandwich structure can be controlled by an external electric/magnetic field in a reversible manner. Sandwich structures with ER/MR material are known to offer attractive potentials for vibration attenuation over a wide range of frequencies and temperatures [6, 7. Moreover, additive manufacturing such as 3D printing allows for the design of complex core materials, honeycomb filled with a MR elastomer for instance [ 8 , improving the mechanical stability, stiffness and energy absorption of the structure. Recent state-of-the-art reviews are discussed in 9 -11].

As for VE models, classical ER/MR models combine elastic springs and viscous dashpots to describe the rheological properties. These can be solid-like models such as Kelvin-Voigt and Zener elements model, and fluid-models such as Maxwell fluid and three-parameter fluid model [12. Rheological parameters are characterized from experiments carried out on ER/MR fluids or elastomers [9]. The shear modulus $G_{c}^{*}$ can be written as a function of the driving frequency $\omega$ and the varying intensity $p$ of the applied field over some interval $I$ :

$$
G_{c}^{*}(\omega, p)=G_{c}(\omega, p)\left[1+i \eta_{c}(\omega, p)\right],
$$

where the real part $G_{c}(\omega, p)$ is the storage modulus and the ratio $\eta_{c}(\omega, p)$ represents the loss factor of the core material. The imaginary part $G_{c}(\omega, p) \eta_{c}(\omega, p)$ is the loss modulus. The rheological law (1) may be implemented in the finite element method (FEM) to predict the dynamic behaviour of a sandwich structure [12 15] or to study its sensitivity with respect to modelling parameters such as thickness ratio, material properties or field intensity values [16 21]. Note that most of the finite element analyses (FEA) assume a constant frequency shear modulus.

When a frequency dependent core is embedded in a structure, the free vibration analysis based on FEM can be formulated as the generalised nonlinear eigenvalue problem:

$$
\left[\mathbf{K}(\omega(p), p)-\omega^{2}(p) \mathbf{M}(p)\right] u(p)=0
$$

where $\mathbf{K}$ is the stiffness matrix, $\mathbf{M}$ is the mass matrix and $(u(p), \omega(p))$ is the eigensolution. Structural damping properties $(\Omega(p), \eta(p))$ are deduced following:

$$
\omega^{2}(p)=\Omega^{2}(p)(1+i \eta(p)), \quad \text { for } p \in I,
$$

where $\Omega(p)$ is the resonant frequency and $\eta(p)$ is the structural loss factor. For a prescribed parameter $p$, the nonlinear frequency dependent problem (2) can be solved 
as proposed in 22], for instance. This algorithm combines homotopy to a higher order continuation method and agrees with the VE rheological modelling. Intensity effects can be thus evaluated at given values $p$ of the interval.

The calculation of continuous approximations for $(u(p), \omega(p))$ should take into account the continuous variation of the modelling parameter $p \in I$. A very few methods propose an actual continuous approach in their parametric analyses. Among them, He 23] presents some analytical perturbation and homotopy analytical techniques for the solution of nonlinear differential equations by scaling the modelling parameter $p$ by the homotopy parameter. Likewise, Charpentier and Lampoh 24 combine FEM, homotopy and higher order continuation to analyse the sensitivity of eigensolutions with respect to a damping function scaled by the homotopy parameter. Building on previous works [22, 25] 27], Akoussan et al [28, 29] propose a higher order continuation method for the sensitivity analysis of the damping properties of frequency dependent VE composite multilayer plates with respect to geometrical modelling parameters such as the layer thickness ratio and the fibers' orientation, respectively.

The calculation of the vibration behaviour over a large frequency range and/or a large field intensity range is invaluable from a design point of view to determine maximum damping configurations. To the best of our knowledge, any method has been proposed to evaluate the damping capabilities over a continuous range of electric/magnetic field intensity. The homotopy-based numerical method we present builds on 22 to fill this gap.

The manuscript is organized as follow. Section 2 presents the nonlinear free vibration problem associated to the FEM. The two-step homotopy method proposed for the continuous vibration analysis is described with detail in section 3 Numerical results, carried out on ER/MR beams and plates published in the literature, are discussed in section 4. Section 5 provides concluding remarks and future perspectives.

\section{Setting of the residual nonlinear problem}

For the sake of generality [22], the nonlinear eigenvalue problem (2) is written in the residual form:

$$
\mathcal{R}(u(p), \lambda(p), p)=[\mathbf{K}(\lambda(p), p)-\lambda(p) \mathbf{M}(p)] u(p)=0,
$$

where $u(p)=u \in \mathbb{C}^{N}$ denotes the eigenmode, $\lambda(p)=\lambda \in \mathbb{C}$ is the eigenvalue such that $\lambda=\omega^{2}$, and $p \in I=\left[p_{\min }, p_{\max }\right]$ represents the modelling parameter. In the paper, the modelling parameter $p$ to be varied continuously is the intensity of the electric or magnetic field.

The residual problem (4) can be written in a simplified manner as:

$$
\mathcal{R}(u, \lambda, p)=[\mathbf{K}(\lambda, p)-\lambda \mathbf{M}] u=0,
$$

since the mass matrix of the ER/MR sandwich structure does not depend on the applied field.

The stiffness matrix $\mathbf{K}(\lambda, p)$ is decomposed into:

$$
\mathbf{K}(\lambda, p)=\mathbf{K}_{\mathbf{0}}^{*}+G^{*}(\lambda, p) \mathbf{K}_{\mathbf{v}},
$$

where $\mathbf{K}_{\mathbf{0}}^{*}=\mathbf{K}(0,0)$ is the finite element delayed elasticity stiffness matrix computed assuming $\lambda=0$ and $p=0, \mathbf{K}_{\mathbf{v}}$ is a real matrix and $G^{*}(\lambda, p)$ is a complex nonlinear scalar function describing the dependence of the stiffness matrix in both the frequency 
and the field intensity. Introducing (6) into (5) yields the nonlinear general eigenvalue problem:

$$
\mathcal{R}(u, \lambda, p)=\left[\mathbf{K}_{\mathbf{0}}^{*}+G^{*}(\lambda, p) \mathbf{K}_{\mathbf{v}}-\lambda \mathbf{M}\right] u=0 .
$$

This nonlinear problem cannot be solved in a straightforward manner, even for a prescribed value of the field intensity $p$. The reader is referred to [30] for a review of nonlinear eigenvalue problems.

\section{Generalised homotopy}

Former studies [22, 31] propose a continuous method for the solution of nonlinear eigenvalue problems. The main idea is to split the residual problem (7) into an eigenvalue problem $\mathcal{S}(u, \lambda)=0$ with known solutions and some additional nonlinear contribution $\mathcal{T}(u, \lambda, p)$, that is:

$$
\mathcal{R}(u, \lambda, p)=\mathcal{S}(u, \lambda)+\mathcal{T}(u, \lambda, p)=0,
$$

where the functions $\mathcal{S}$ and $\mathcal{T}$ are specific to the problem under study (see table 1) and case studies presented in section 4 .

As described in subsection 3.1 , the solutions of $\mathcal{S}(u, \lambda)=0$ can be then deformed to compute the solutions of $\mathcal{R}(u, \lambda)=0$ through the so-called homotopy method.

The generalized higher order homotopy method we propose moreover allows for the continuous solution of the nonlinear eigenvalue problem while conserving its mechanical/physical meaning. In others words, the continuous variations of some material or geometrical or intensity parameter $p$ onto the eigenvalues $(u(p), \lambda(p))$, resonant frequencies $\Omega(p)$ or loss factors $\eta(p)$ can be studied in an actual continuous manner.

The use and benefits of this new generalized homotopy-based eigensolver are exemplified in subsection 3.2 and section 4

\subsection{General nonlinear eigensolver}

The solution of (8) is implemented through a homotopy method mapping $\mathcal{S}$ to $\mathcal{R}$ by computing continuous branches of solutions for:

$$
\mathcal{R}(u(\alpha), \lambda(\alpha), p(\alpha))=\mathcal{S}(u, \lambda)+\alpha \mathcal{T}(u, \lambda, p)=0,
$$

for the homotopy parameter $\alpha \in \mathbb{R}$ varying in the range $\left[\alpha_{\min }, \alpha_{\max }\right]$.

The unknowns are developed into Taylor series in the vicinity of $\alpha$ to be able to evaluate continuous approximation branches:

$$
\begin{aligned}
& \lambda\left(\alpha+\delta_{\alpha}, p\right) \approx \sum_{n=0}^{N} \frac{\left(\delta_{\alpha}\right)^{n}}{n !} \frac{\partial^{n} \lambda}{\partial \alpha^{n}}(\alpha, p)=\sum_{n=0}^{N}\left(\delta_{\alpha}\right)^{n} \lambda_{n}, \\
& u\left(\alpha+\delta_{\alpha}, p\right) \approx \sum_{n=0}^{N} \frac{\left(\delta_{\alpha}\right)^{n}}{n !} \frac{\partial^{n} u}{\partial \alpha^{n}}(\alpha, p)=\sum_{n=0}^{N}\left(\delta_{\alpha}\right)^{n} u_{n},
\end{aligned}
$$

where $\delta_{\alpha}$ is a small perturbation of $\alpha$ and $N$ is the truncation order. Unknowns $u_{n}$ and $\lambda_{n}$ are the Taylor coefficients of $u$ and $\lambda$, respectively.

As noted in [23, 24], the linear variation of the modelling parameter $p$ can be accounted by scaling it with respect to the homotopy parameter $\alpha \in \mathbb{R}$. However, 
see section 4 most of the ER/MR constitutive laws cannot be reformulated to exhibit such a linear dependence.

Any nonlinear bijective function mapping $p$ to $\alpha$, for instance $F(p(\alpha))=\alpha$ and $F^{-1}(\alpha)=p$, can be accounted through a higher order differentiation of $F(p)$ with respect to the homotopy parameter $\alpha$. This is implemented by means of the chain rule and the Taylor expansion of $p(\alpha)$ with respect to $\alpha$ :

$$
p\left(\alpha+\delta_{\alpha}\right) \approx \sum_{n=0}^{N} \frac{\left(\delta_{\alpha}\right)^{n}}{n !} \frac{\partial^{n} p}{\partial \alpha^{n}}(\alpha)=\sum_{n=0}^{N}\left(\delta_{\alpha}\right)^{n} p_{n},
$$

where $p_{n}$ are the Taylor coefficients of $p$. From a practical point of view, the Taylor series (12) implements the variation of ER/MR properties parametrized by $p$. The higher order approximations $(10)-(12)$ then allow for the calculation of continuous branches of solutions with respect to the variation of $p$ in the range $\left[p_{\min }, p_{\max }\right]$.

Introducing these series into (9) yields:

$$
\mathcal{R}\left(u\left(\alpha+\delta_{\alpha}\right), \lambda\left(\alpha+\delta_{\alpha}\right), p\left(\alpha+\delta_{\alpha}\right)\right) \approx \sum_{n=0}^{N}\left(\delta_{\alpha}\right)^{n} \mathcal{R}_{n}=0,
$$

where $\mathcal{R}_{n}$ are the Taylor coefficients of $\mathcal{R}$. The analyticity assumption implies:

$$
\mathcal{R}_{n}=\mathcal{S}_{n}+\alpha \mathcal{T}_{n}=0 \quad \text { for } n=1, . ., N,
$$

where the Taylor coefficients $\mathcal{S}_{n}$ and $\mathcal{T}_{n}$ of functions $\mathcal{S}$ and $\mathcal{T}$ satisfy:

$$
\mathcal{S}\left(u\left(\alpha+\delta_{\alpha}\right), \lambda\left(\alpha+\delta_{\alpha}\right)\right) \approx \sum_{n=0}^{N}\left(\delta_{\alpha}\right)^{n} \mathcal{S}_{n},
$$

and

$$
\mathcal{T}\left(u\left(\alpha+\delta_{\alpha}\right), \lambda\left(\alpha+\delta_{\alpha}\right), p\left(\alpha+\delta_{\alpha}\right)\right) \approx \sum_{n=0}^{N}\left(\delta_{\alpha}\right)^{n} \mathcal{T}_{n} .
$$

Faà di Bruno's generalised chain rule formula 22 allows to write a sequence of $N+1$ equations for the unknown Taylor coefficients $u_{n}$ and $\lambda_{n}$.

At order $n=0$, equation (14) is restricted to:

$\mathcal{J} u_{0}=\left(\left\{\left.\mathcal{S}_{1}\right|_{u_{1}=I d}, \lambda_{1}=0\right\}+\alpha_{(j)}\left\{\left.\mathcal{T}_{1}\right|_{u_{1}=I d}, \lambda_{1}=0, p_{1}=0\right\}\right) u_{0}=0$,

where $\mathcal{J},\left\{\left.\mathcal{S}_{1}\right|_{u_{1}=I d}, \lambda_{1}=0\right\}$ and $\left\{\left.\mathcal{T}_{1}\right|_{u_{1}=I d}, \lambda_{1}=0\right\}$ are Jacobian matrices, differentiated with respect to $u$. One deduces that ${ }^{t} u_{0} \mathcal{J}=0$ since the finite element matrices contained in $\mathcal{J}$ are symmetric.

At order $n$, equation (14) is split into some first order terms multiplied by the unknown Taylor coefficients $u_{n}$ and $\lambda_{n}$ and some complementary higher order terms. One deduces:

$$
\begin{aligned}
& \left(\left\{\left.\mathcal{S}_{1}\right|_{u_{1}=I d}, \lambda_{1}=0\right\}+\alpha_{(j)}\left\{\left.\mathcal{T}_{1}\right|_{u_{1}=I d}, \lambda_{1}=0, p_{1}=0\right\}\right) u_{n}+ \\
& +\left(\left\{\left.\mathcal{S}_{1}\right|_{u_{1}=0, \lambda_{1}=1}\right\}+\alpha_{(j)}\left\{\left.\mathcal{T}_{1}\right|_{u_{1}=0, \lambda_{1}=1, p_{1}=0}\right\}\right) \lambda_{n}+ \\
& +\left\{\left.\mathcal{S}_{n}\right|_{u_{n}=0, \lambda_{n}=0}\right\}-\alpha_{(j)}\left\{\left.\mathcal{T}_{n}\right|_{u_{n}=0, \lambda_{n}=0}\right\}-\mathcal{T}_{n-1}=0 .
\end{aligned}
$$


For the sake of clarity, 20 is rewritten as:

$$
\mathcal{J} u_{n}+\mathcal{D} \lambda_{n}+\mathcal{H}^{n}=0
$$

where the gradient vector $\mathcal{D}$, differentiated with respect to $u$ and $\lambda$, satisfies:

$$
\mathcal{D}=\left\{\left.\mathcal{S}_{1}\right|_{u_{1}=0, \lambda_{1}=1}\right\}+\alpha\left\{\left.\mathcal{T}_{1}\right|_{u_{1}=0, \lambda_{1}=1, p_{1}=0}\right\} .
$$

The nonlinear higher order contribution at order $n$ is:

$$
\mathcal{H}^{n}=\left\{\left.\mathcal{S}_{n}\right|_{u_{n}=0, \lambda_{n}=0}\right\}+\alpha\left\{\left.\mathcal{T}_{n}\right|_{u_{n}=0, \lambda_{n}=0}\right\}+\mathcal{T}_{n-1} .
$$

Multiplying (21) by ${ }^{t} u_{0}$ yields the following equation for the unknown $\lambda_{n}$ :

$$
\lambda_{n}=-\frac{{ }^{t} u_{0} \mathcal{H}^{n}}{{ }^{t} u_{0} \mathcal{D}} .
$$

Equation 21) cannot be solved in a straightforward manner since the Jacobian $\mathcal{J}$ is singular. The orthogonality condition (25) is added to close the system:

$$
{ }^{t} u_{0}\left(u(\alpha)-u_{0}\right)=0 \text {. }
$$

It implies that the eigenmode $u_{0}$ is orthogonal to the Taylor coefficients $u_{n}$ of $u$ for $n=1, . ., N$.

A Lagrange multiplier $\kappa$ is introduced [31] to compute $u_{n}$ following:

$$
\left[\begin{array}{cc}
\mathcal{J} & u_{0} \\
{ }^{t} u_{0} & 0
\end{array}\right]\left\{\begin{array}{c}
u_{n} \\
\kappa
\end{array}\right\}=\left\{\begin{array}{c}
-\mathcal{D} \lambda_{n}-\mathcal{H}^{n} \\
0
\end{array}\right\} .
$$

The coefficients of the series $(10)$ and $(11)$ are obtained by computing the higher order term $\mathcal{H}^{n}$, by evaluating (24) and by solving the linear system of (26), in an alternate manner.

The convergence radius $\alpha^{*}$ of the computed series is estimated as:

$$
\alpha^{*}=\left(\varepsilon \frac{\left\|u_{1}\right\|}{\left\|u_{N}\right\|}\right)^{\frac{1}{N-1}}
$$

where $\varepsilon$ is a small parameter (usually $\varepsilon=10^{-6}$ ). A branch of solutions is then built from Taylor expansions of $u$ and $\lambda$ by evaluating the series up to $\alpha^{*}$.

For each mode, one may deduce Taylor expansions for the resonant frequency $\Omega^{2}(p)$ and the loss factor $\eta(p)$ thanks to the chain rule since they satisfy (3). Two important benefits can be highlighted. Firstly, continuous branches of solutions $\left(p, \Omega^{2}(p)\right)$ and $(p, \eta(p))$ can be plotted as presented in section 4 for different structures and constitutive laws. Secondly, Taylor expansions can be used to determine minimum or maximum values in a range in a very efficient and accurate manner.

The computation of an additional solution branch is required as far as $\alpha_{\max }$ is not reached. The new branch is computed from the solution of the previous branch by setting $\left(u_{0}, \lambda_{0}\right)=\left(u\left(\alpha^{*}\right), \lambda\left(\alpha^{*}\right)\right)$.

Note that all the derivative computation are carried out by means of automatic differentiation. 32. This provides generality to the nonlinear continuous eigensolver. 


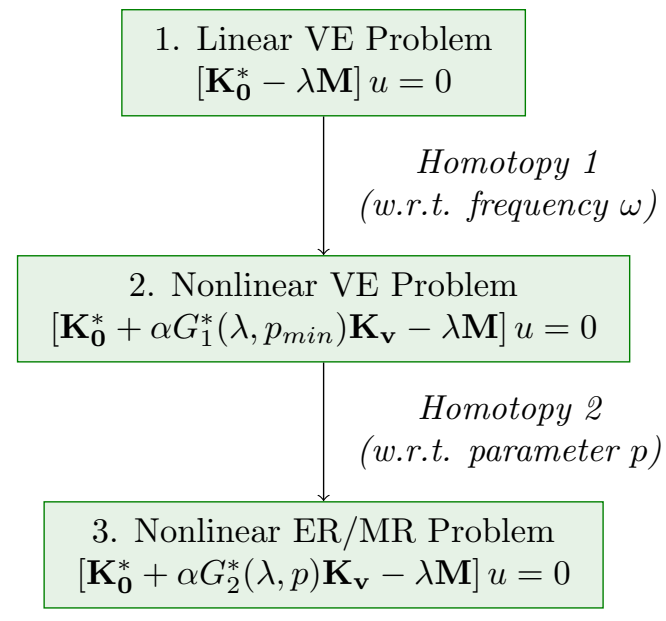

Figure 1. Structure of the general eigensolver for nonlinear continuous ER or MR eigenproblems.

Table 1. Input data for the homotopies carried out with respect to the frequency $\omega$ and the field intensity $p$, respectively.

\begin{tabular}{lll}
\hline & $\begin{array}{l}\text { Homotopy } 1 \\
(\text { w.r.t. frequency } \omega)\end{array}$ & $\begin{array}{l}\text { Homotopy } 2 \\
(\text { w.r.t. parameter } p)\end{array}$ \\
\hline $\mathcal{S}(u, \lambda)$ & {$\left[\mathbf{K}_{\mathbf{0}}^{*}-\lambda \mathbf{M}\right] u$} & {$\left[\mathbf{K}_{\mathbf{0}}^{*}+G_{1}^{*}\left(\lambda, p_{\text {min }}\right) \mathbf{K}_{\mathbf{v}}-\lambda \mathbf{M}\right] u$} \\
$\mathcal{T}(u, \lambda, p)$ & $G_{1}^{*}\left(\lambda, p_{\min }\right) \mathbf{K}_{\mathbf{v}} u$ & $G_{2}^{*}(\lambda, p) \mathbf{K}_{\mathbf{v}} u$ \\
$\alpha$ & $\alpha \in \mathbb{R}$ & $F(p)$ \\
$p$ & $p_{\min }$ & $F^{-1}(\alpha)=\sum_{n=0}^{N}\left(\delta_{\alpha}\right)^{n} p_{n}$ \\
$\alpha_{\min }$ & 0 & $F\left(p_{\min }\right)$ \\
$\alpha_{\max }$ & 1 & 1 \\
$G^{*}(\lambda, p)$ & $G_{1}^{*}\left(\lambda, p_{\min }\right)$ & $G_{2}^{*}(\lambda, p)$ \\
\hline
\end{tabular}

\subsection{Specialisation for $E R / M R$ law}

The calculation of the vibration behaviour of an ER/MR sandwich structure over a continuous range $\left[p_{\min }, p_{\max }\right]$ of electric/magnetic field intensity can be carried out as follows.

The general homotopy process described in subsection 3.1 is now specialised to agree with the algorithm described in figure 1. Therein, the homotopy solver is run twice from input data (parameters, parameter ranges, rheological laws and functions) reported in table 1 .

The first homotopy is the one described in 22. It deforms continuously the 
solution of the linear VE problem:

$$
\left[\mathbf{K}_{\mathbf{0}}^{*}-\lambda \mathbf{M}\right] u=0,
$$

related to $\alpha=0$ and computable using a classical eigensolver, to the solution for the nonlinear VE problem:

$$
\left[\mathbf{K}_{\mathbf{0}}^{*}+\alpha G_{1}^{*}(\lambda, p) \mathbf{K}_{\mathbf{v}}-\lambda \mathbf{M}\right] u=0, \quad \text { for } \alpha \in[0,1],
$$

where the parameter $p$ is temporarily set equal to $p_{\text {min }}$, as written in table1. However, in the vibration analysis of a VE sandwich structure [22, one is interested in this last solution only.

The second homotopy is devoted to the evaluation of the continuous variation of the eigensolution with respect to the modelling parameter $p$. Its input data are chosen to map the field intensity parameter $p \in\left[p_{\min }, p_{\max }\right]$ to the homotopy parameter $\alpha$ through the bijective scaling function $\alpha=F(p)$. The homotopy parameter thus varies in $\left[F\left(p_{\min }\right), 1\right]$.

Any actual continuous rheological law $G_{1}^{*}(\lambda, p)$ can be accounted. It suffices to split it into some real-valued bijective scaling function $F(p)$ and a complex-valued virtual law $G_{2}^{*}(\lambda, p)$ satisfying:

$$
G_{1}^{*}(\lambda, p)=F(p) G_{2}^{*}(\lambda, p) .
$$

The scaling function $F(p) \in \mathbb{R}$ can be identified to the homotopy parameter $\alpha \in \mathbb{R}$ as far as it satisfies $F\left(p_{\max }\right)=\alpha_{\max }=1$.

For efficiency reasons, it is convenient to choose $F$ so that its reciprocal function $F^{-1}$ is of simple computation. Where possible, one may also choose $G_{2}^{*}$ independent from $p$. Typically, depending on the law, one may choose either $F(p)=p / p_{\max }$ or $F(p)$ as a polynomial fraction of $p$. Note that the choice of the function $F$ may impact the number of iterations and the distance between two consecutive iterations.

When the constitutive law is independent from the frequency, it is worth mentioning that the first homotopy can be replaced by a complex eigenvalue solver applied to the linear VE problem.

\section{Numerical results}

The vibration analysis is carried out on three ER or MR sandwich structures described in the literature to assess the benefits of our continuous homotopy approach over the classical incremental approaches.

From a numerical point of view, the eigenvalue problems are discretized using the finite element method. The number of elements is specified for each case study in the tables describing the geometrical and material properties. The truncature order of the Taylor expansions is $N=20$. With regards to formula (27), the convergence radius $a_{\max }$ and the parameter $\varepsilon$ are both set equal to $10^{-6}$. These data allow for reproducibility.

4.1. Rectangular "Aluminium/ER fluid/Aluminium" sandwich plate with a frequency independent constitutive law

The rectangular sandwich plate 33 presented in figure 2 comprises a lower host plate (layer 3 ) and an upper constraining plate (layer 1) made of aluminium alloy, and a core (layer 2) composed of an outer adhesive joint embedding an ER fluid. This study is 

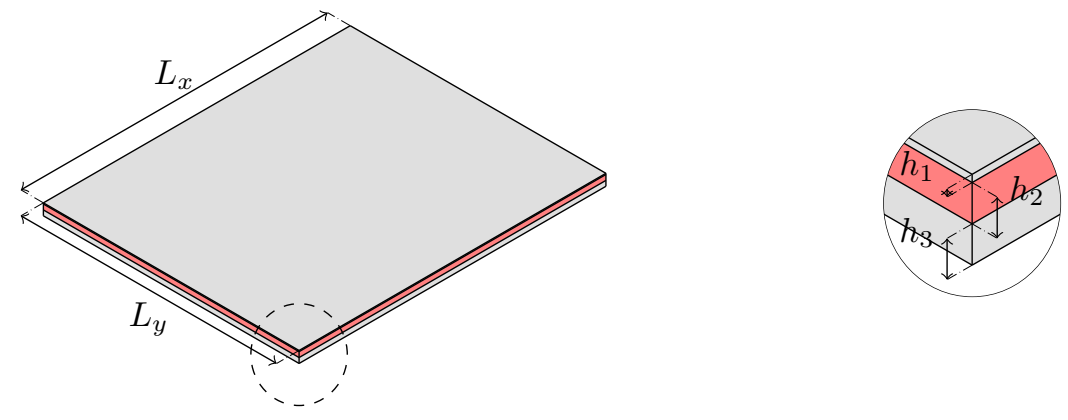

Figure 2. Rectangular "Aluminium/ER fluid/Aluminium" sandwich plate described in 33 .

based on the four-node plate element used in 34. In the FEM, the core properties are assumed to be homogeneous. This plate is simply supported on all its edges (SSSS).

The geometrical and material properties of the structure are reported in table 2 . The shear modulus of the core material $G_{c}^{*}(E)$, as measured in 35, depends on the electric field intensity $E$ ranging from $0 \mathrm{kV} \cdot \mathrm{mm}^{-1}$ to $3.5 \mathrm{kV} \cdot \mathrm{mm}^{-1}$ :

$$
\begin{aligned}
& G_{c}^{*}(E)=15000 E^{2}+i 6900, \\
& G_{c}^{*}(E)=G_{0}^{*}+G_{1}^{*}(E) .
\end{aligned}
$$

As noted in subsection 3.2 , the first homotopy is useless since the constitutive law (31) does not depend on frequency.

For each eigenmode of the VE problem, the continuous variation of the nonlinear eigenvalue with respect to the field intensity is operated by mean of the "second" homotopy.

The law (31) is here split into a constant part $G_{0}^{*}=G_{c}^{*}(0)=i 6900$ and a nonlinear part $G_{1}^{*}(E)=15000 E^{2}$. Following the discussion proposed in subsection 3.2 , the nonlinear part of $(32)$ is written as:

$$
G_{1}^{*}(E)=F(E) G_{2}^{*}(E)=\alpha G_{2}^{*}(E) .
$$

The scaling function $F(E)=\alpha$ can be chosen as:

$$
F(E)=\frac{E^{2}}{E_{\max }^{2}}=\alpha,
$$

then $G_{2}^{*}(E)$ is equal to $15000 E_{\max }^{2}$. Even other choices are possible for the function $F(E)$, (34) is the simplest one that describes the range of interest $\left[0 \mathrm{kV} \cdot \mathrm{mm}^{-1}, 3.5 \mathrm{kV} \cdot \mathrm{mm}^{-1}\right]$ since it yields the independence of $G_{2}^{*}$ with respect to both the frequency and the electric field.

The nonlinear continuous branches of the first four natural frequencies and modal loss factors of the ER rectangular sandwich plate are plotted in figure 3 for a comparison with the discrete natural frequencies and modal loss factors computed by the incremental approach used in [33. The agreement at the discrete points obtained with the incremental method is very good. Minor discrepancies (less than 6\%) can be observed in table 3 for both resonance frequencies and loss factors. These result from different finite element discretization. Moreover, in the kinematic assumptions, the normal stress is accounted in the core layer in our FEM while it is neglected in the FEM implemented in 33 . 
Table 2. Geometrical and material properties of the rectangular "Aluminium/ER fluid/Aluminium" plate described in [33].

\begin{tabular}{lll}
\hline Plate & Length & $L_{x}=300 \mathrm{~mm}$ \\
& Width & $L_{y}=250 \mathrm{~mm}$ \\
& Finite element mesh & $60 \times 50$ elements \\
Elastic faces & Young modulus & $E_{1}=E_{3}=7 \times 10^{10} \mathrm{~Pa}$ \\
& Poisson ratio & $\nu_{1}=\nu_{3}=0.29$ \\
& Density & $\rho_{1}=\rho_{3}=2700 \mathrm{~kg} \cdot \mathrm{m}^{-3}$ \\
& Thickness & $h_{1}=0.05 \mathrm{~mm}$ \\
& & $h_{3}=0.5 \mathrm{~mm}$ \\
ER fluid & Shear modulus & $G_{c}^{*}(E)=i 6900+15000 E^{2}$ \\
& Poisson ratio & $\nu_{2}=0.5$ \\
& Density & $\rho_{2}=1700 \mathrm{~kg} \cdot \mathrm{m}^{-3}$ \\
& Thickness & $h_{2}=0.5 \mathrm{~mm}$ \\
\hline
\end{tabular}

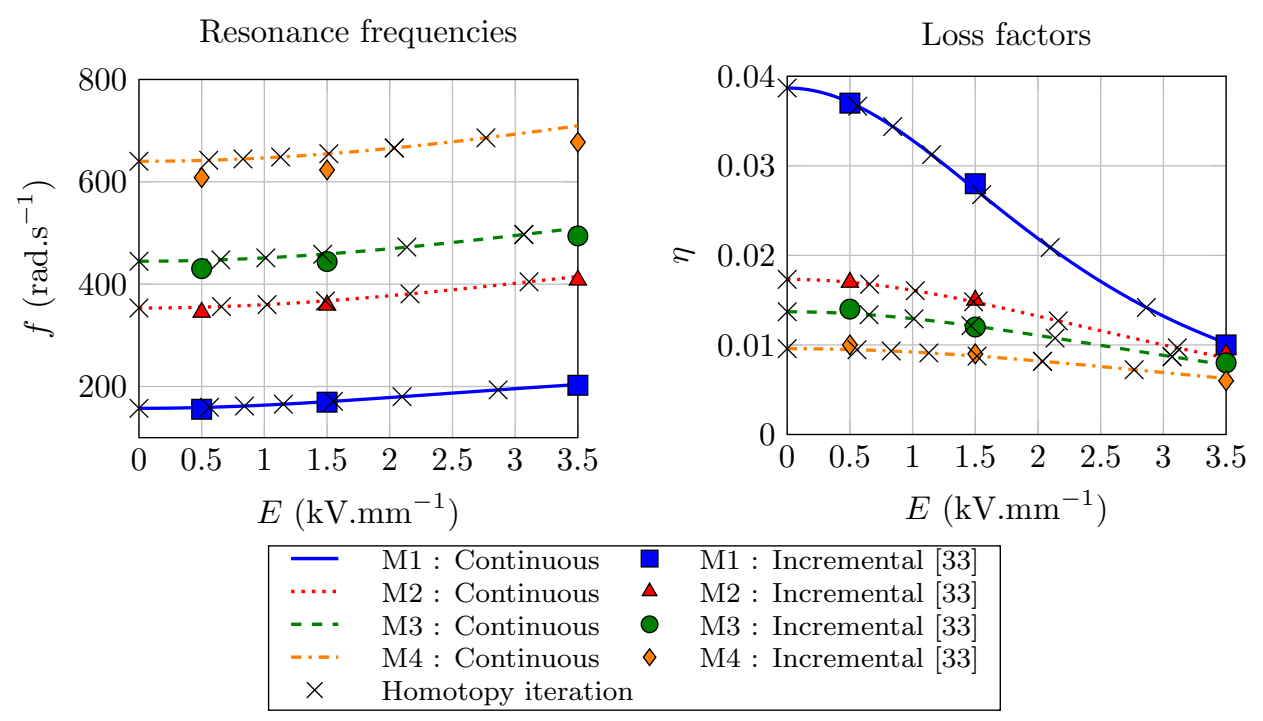

Figure 3. Rectangular "Aluminium/ER fluid/Aluminium" sandwich plate. Comparison of the first four resonance frequencies and loss factors computed by means of the homotopy method (continuous solid/dashed or dotted lines) and the incremental methods (coloured markers).

Note that points where series were computed are marked with $\mathrm{a} \times$ to indicate the number of iterations. One observes that 7 series computations are needed to plot the variation of the first natural frequency $f$ with respect to the variation of the electric field intensity $E$.

\subsection{Rubber sealed "Aluminium/MR fluid/Aluminium" sandwich beam with a frequency independent constitutive law}

The rectangular sandwich beam [36] presented in figure 4 is studied under clamped-free (CF) boundary conditions. This study is based on the two-node beam element used in 37. The external elastic face layers (layers 1 and 3) are made of aluminium alloy 
Continuous eigenvalue solver for the design of ER/MR sandwich structures

Table 3. Rectangular "Aluminium/ER fluid/Aluminium" sandwich plate. Comparison of the first four resonance frequencies, loss factors and relative errors.

\begin{tabular}{lllllll}
\hline$E\left(\mathrm{kV} . \mathrm{mm}^{-1}\right)$ & $\begin{array}{l}\text { Homotopy } \\
\text { method }\end{array}$ & \multicolumn{3}{c}{$\begin{array}{l}\text { Incremental } \\
\text { method }[36]\end{array}$} & \multicolumn{3}{c}{ Relative error } \\
\cline { 2 - 6 } & $f\left({\left.\mathrm{rad} . \mathrm{s}^{-1}\right)}^{-1}\right.$ & $\eta$ & $f\left(\mathrm{rad}^{-1}\right)$ & $\eta$ & $f(\%)$ & $\eta(\%)$ \\
\hline 0.5 & 159.00 & 0.0371 & 155.53 & 0.037 & 2.23 & 0.27 \\
& 354.98 & 0.0170 & 345.14 & 0.017 & 2.85 & 0.00 \\
& 446.39 & 0.0135 & 430.36 & 0.014 & 3.72 & 3.57 \\
& 641.72 & 0.0095 & 608.26 & 0.010 & 5.50 & 5.00 \\
\hline 1.5 & 170.38 & 0.0274 & 169.37 & 0.028 & 0.60 & 2.14 \\
& 367.48 & 0.0148 & 358.99 & 0.015 & 2.36 & 1.33 \\
& 459.02 & 0.0121 & 444.21 & 0.012 & 3.33 & 0.83 \\
& 654.63 & 0.0088 & 623.17 & 0.009 & 5.05 & 2.22 \\
\hline 3.5 & 203.90 & 0.0102 & 202.40 & 0.010 & 0.74 & 2.00 \\
& 415.04 & 0.0086 & 407.99 & 0.009 & 1.73 & 4.44 \\
& 509.81 & 0.0078 & 494.27 & 0.008 & 3.14 & 2.50 \\
& 709.39 & 0.0063 & 677.50 & 0.006 & 4.71 & 5.00 \\
\hline
\end{tabular}

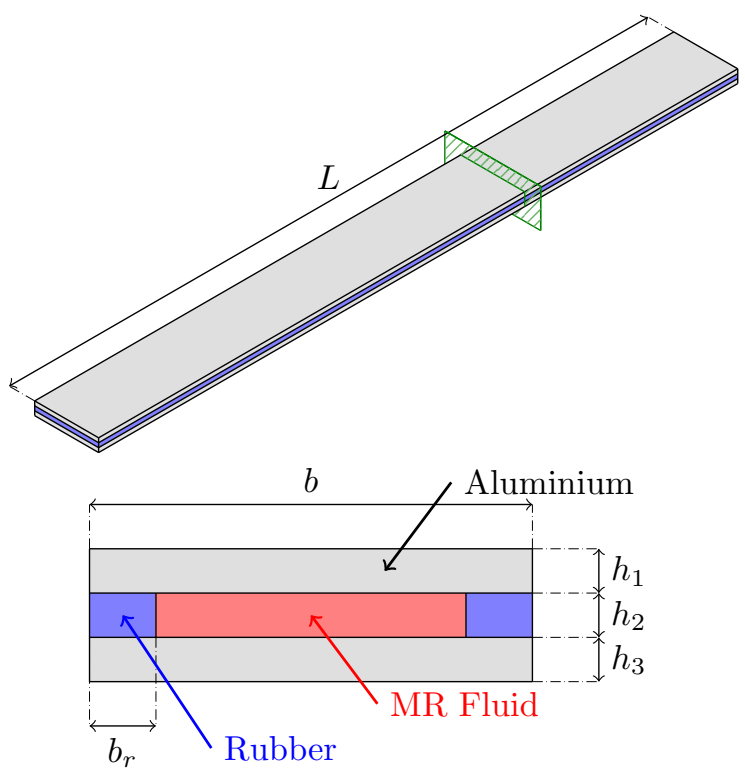

Figure 4. Rubber sealed "Aluminium/MR fluid/Aluminium" sandwich beam described in [36.

and the core layer (layer 2) is made of a MR fluid embedded in a silicon rubber sealant. Their shear moduli are assumed to be independent from the frequency. The magnetic field $B$ is varied from $0 \mathrm{mT}$ to $50 \mathrm{mT}$. The geometrical and material properties of the MR sandwich beam are described in table 4 .

As in [36, the core layer of the sandwich beam comprising the rubber seal and the MR fluid is modelled as a homogeneous material layer. The equivalent shear modulus is expressed as a combination of the moduli and widths of the two materials using the 
Continuous eigenvalue solver for the design of ER/MR sandwich structures

Table 4. Geometrical and material properties of the rubber sealed "Aluminium/MR fluid/Aluminium" sandwich beam described in 36.

\begin{tabular}{lll}
\hline Beam & Length & $L=300 \mathrm{~mm}$ \\
& Width & $b=30 \mathrm{~mm}$ \\
& Finite element mesh & 200 elements \\
Elastic faces & Young modulus & $E_{f}=6.8 \times 10^{10} \mathrm{~Pa}$ \\
& Density & $\rho_{f}=2700 \mathrm{~kg} \cdot \mathrm{m}^{-3}$ \\
& Thickness & $h_{f}=1 \mathrm{~mm}$ \\
MR fluid & Shear modulus & $G_{c}^{*}(B)=G_{0}^{*}+G_{1}^{*}(B)$ \\
& Poisson ratio & $\nu_{c}=0.49$ \\
& Density & $\rho_{c}=3500 \mathrm{~kg} \cdot \mathrm{m}^{-3}$ \\
& Thickness & $h_{c}=1 \mathrm{~mm}$ \\
Rubber sealant & Shear modulus & $G_{r}=1.34 \times 10^{6} \mathrm{~Pa}$ \\
& Width & $b_{r}=1.5 \mathrm{~mm}$ \\
& Density & $\rho_{r}=1233 \mathrm{~kg} \cdot \mathrm{m}^{-3}$ \\
\hline
\end{tabular}

Table 5. Coefficients for the shear modulus formula modelling the viscoelastic behaviour of the MR fluid in the preyield regime (from [36]).

\begin{tabular}{llll}
\hline$a_{0}$ & $8.93 \times 10^{5} \mathrm{~Pa}$ & $b_{0}$ & $1.86 \times 10^{5} \mathrm{~Pa}$ \\
$a_{1}$ & $49975 \mathrm{~Pa} \cdot \mathrm{mT}^{-1}$ & $b_{1}$ & $8124 \mathrm{~Pa}_{\mathrm{mT}} \mathrm{mT}^{-1}$ \\
$a_{2}$ & $-336.91 \mathrm{~Pa} \cdot \mathrm{mT}^{-2}$ & $b_{2}$ & $-90 \mathrm{~Pa} \cdot \mathrm{mT}^{-2}$ \\
\hline
\end{tabular}

so-called "rule of mixture":

$$
G_{c}^{*}(B)=G_{r}\left(\frac{b_{r}}{b}\right)+G_{M R}^{*}(B)\left(1-\frac{b_{r}}{b}\right),
$$

where $b_{r}$ and $b$ are the widths of the rubber and beam respectively, and $G_{r}$ and $G_{c}^{*}$ are the shear modulus of the rubber and MR fluid respectively. In the preyield regime, the MR fluid exhibits a viscoelastic behaviour that can be modelled as [36]:

$$
G_{M R}^{*}(B)=\left(a_{0}+a_{1} B+a_{2} B^{2}\right)+i\left(b_{0}+b_{1} B+b_{2} B^{2}\right),
$$

where the coefficients $a_{i}$ and $b_{i}$ are provided in table 5

The homotopy 1 (see figure 1) is applied by splitting (36) into:

$$
G_{c}^{*}(B)=G_{0}^{*}+G_{1}^{*}(B),
$$

where

$$
G_{0}^{*}=G_{c}^{*}(0)=G_{r}\left(\frac{b_{r}}{b}\right)+\left(a_{0}+i b_{0}\right)\left(1-\frac{b_{r}}{b}\right)
$$

and

$$
G_{1}^{*}(B)=B\left(1-\frac{b_{r}}{b}\right)\left[a_{1}+a_{2} B+i\left(b_{1}+b_{2} B\right)\right] .
$$

As discussed in subsection 3.2 , the shear modulus (37) is split into:

$$
G_{1}^{*}(B)=\alpha G_{2}^{*}(B)
$$

where $\alpha$ is chosen as a simple real-valued scaling function of $B$ such that $F\left(B_{\max }\right)=1$ :

$$
\alpha=F(B)=\frac{B}{B_{\max }} .
$$


Resonance frequencies

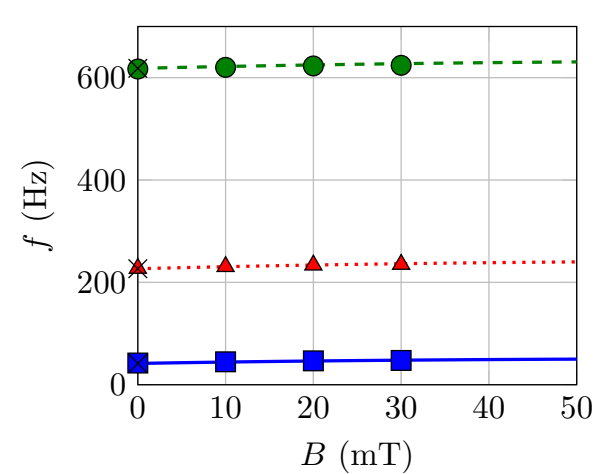

Loss factors

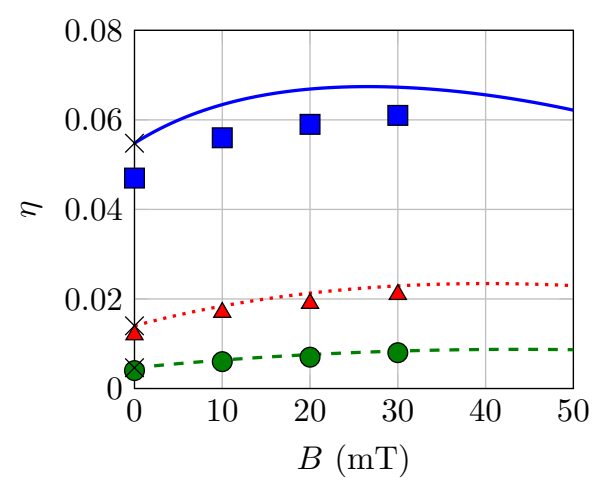

\begin{tabular}{|c|c|c|}
\hline$\longrightarrow$ & M1 : Continuous & M1 : Incremental 36 \\
\hline$\cdots$ & M2 : Continuous & M2 : Incremental 36 \\
\hline $\begin{array}{c}--\cdot \\
x\end{array}$ & M3 : Continuous & M3 : Incremental 36 \\
\hline
\end{tabular}

Figure 5. Rubber sealed "Aluminium/MR fluid/Aluminium" sandwich beam. Comparison of the first three resonance frequencies and loss factors computed by means of the homotopy method (continuous solid/dashed or dotted lines) and the incremental methods (coloured markers).

The complex-valued and field dependent function $G_{2}^{*}(B)$ then satisfies:

$$
G_{2}^{*}(B)=B_{\max }\left(1-\frac{b_{r}}{b}\right)\left[a_{1}+a_{2} B+i\left(b_{1}+b_{2} B\right)\right] .
$$

Under these assumptions, the nonlinear eigenproblem $(7)$ is quadratic 30 in the field intensity $B$.

Figure 5 plots the nonlinear branches of resonant frequencies and modal loss factors computed in the full range of validity $[0 \mathrm{mT}, 50 \mathrm{mT}]$ of the rheological law using continuous solid, dashed and dotted lines. Continuous branches of solutions presented in figure 5 reflect the nonlinearity of the constitutive law with respect to the magnetic field intensity. Note that a unique branch of solutions suffices to describe the specified range of intensity because the range of validity of the series is here larger than $50 \mathrm{mT}$ Resonant frequencies and loss factors computed for 0, 10, 20 and $30 \mathrm{mT}$ by means of the incremental approach [36] are plotted as well.

A decay in the loss factor of the first mode is observed for larger $B$. The incremental method does not exhibit this decay because too few points were reported in 36. A dichotomy method applied to the Taylor series of the loss factor of the first mode then indicates that the maximal damping occurs near to $B \simeq 26.17 \mathrm{mT}$.

Numerical results are presented in table 6. A very good agreement with an error less than $2 \%$ is obtained between the resonant frequencies. A good agreement and similar trends are observed for the loss factors. One observes that the discrepancies on the loss factors are of the same order for the first three modes. These are mainly due to differences in the kinematic modelling. For instance, our model does not include the shearing effect in the face layers. 
Continuous eigenvalue solver for the design of ER/MR sandwich structures

Table 6. Rubber sealed "Aluminium/MR fluid/Aluminium" sandwich beam. Comparison of the first five resonance frequencies, loss factors and relative errors.

\begin{tabular}{lllllll}
\hline$B(\mathrm{mT})$ & \multicolumn{2}{l}{$\begin{array}{l}\text { Homotopy } \\
\text { method }\end{array}$} & \multicolumn{3}{l}{$\begin{array}{l}\text { Incremental } \\
\text { method }\end{array}$} \\
\cline { 2 - 7 } & $f(\mathrm{~Hz})$ & $\eta$ & $f(\mathrm{~Hz})$ & $\eta$ & \multicolumn{2}{c}{ Relative error } \\
\hline 0 & 41.67 & 0.0548 & 42.38 & 0.047 & 1.68 & 16.60 \\
& 227.42 & 0.0140 & 227.44 & 0.012 & 0.01 & 16.67 \\
& 620.44 & 0.0046 & 616.92 & 0.004 & 0.57 & 15.00 \\
& 1207.70 & 0.0022 & 1196.1 & 0.002 & 0.97 & 10.00 \\
& 1990.22 & 0.0013 & 1962.9 & 0.001 & 1.39 & 30.00 \\
\hline 10 & 44.24 & 0.0634 & 44.78 & 0.056 & 1.21 & 13.21 \\
& 231.14 & 0.0184 & 230.95 & 0.017 & 0.08 & 8.24 \\
& 623.85 & 0.0063 & 620.15 & 0.006 & 0.60 & 5.00 \\
& 1210.93 & 0.0031 & 1199.1 & 0.003 & 0.99 & 3.33 \\
& 1993.39 & 0.0019 & 1965.9 & 0.002 & 1.40 & 5.00 \\
\hline 20 & 46.22 & 0.0669 & 46.65 & 0.059 & 0.92 & 13.39 \\
& 234.22 & 0.0213 & 233.87 & 0.019 & 0.15 & 12.11 \\
& 626.74 & 0.0076 & 622.88 & 0.007 & 0.62 & 8.57 \\
& 1213.73 & 0.0038 & 1201.8 & 0.003 & 0.99 & 26.67 \\
& 1996.09 & 0.0022 & 1968.5 & 0.002 & 1.40 & 10.00 \\
\hline 30 & 47.73 & 0.0673 & 47.42 & 0.061 & 0.65 & 10.33 \\
& 236.70 & 0.0229 & 235.24 & 0.021 & 0.62 & 9.05 \\
& 629.12 & 0.0084 & 624.13 & 0.008 & 0.80 & 5.00 \\
& 1216.02 & 0.0042 & 1203.9 & 0.004 & 1.01 & 5.00 \\
& 1998.33 & 0.0025 & 1970.6 & 0.002 & 1.41 & 25.00 \\
\hline & & & & & &
\end{tabular}

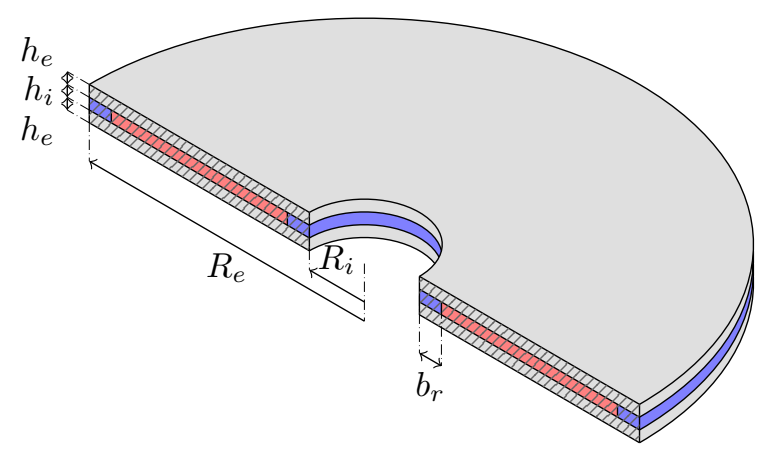

Figure 6. Rubber sealed "Aluminium/MRF 132DG/Aluminium" annular sandwich plate.

\subsection{Rubber sealed "Aluminium/MRF 132DG/Aluminium" annular sandwich plate} with a frequency dependent constitutive law

The annular sandwich plate 9 presented in figure 6 comprises two external elastic layers made of aluminium alloy (layers 1 and 3) and a MR fluid core enclosed in a silicon rubber sealant (layer 2). The geometrical and material properties of the structure are reported in table 7 . The annular plate is free at its inner edge and simply supported at its outer edge (FS). This study is based on the four-node plate element used in 34 with a radial mesh. 
Continuous eigenvalue solver for the design of ER/MR sandwich structures

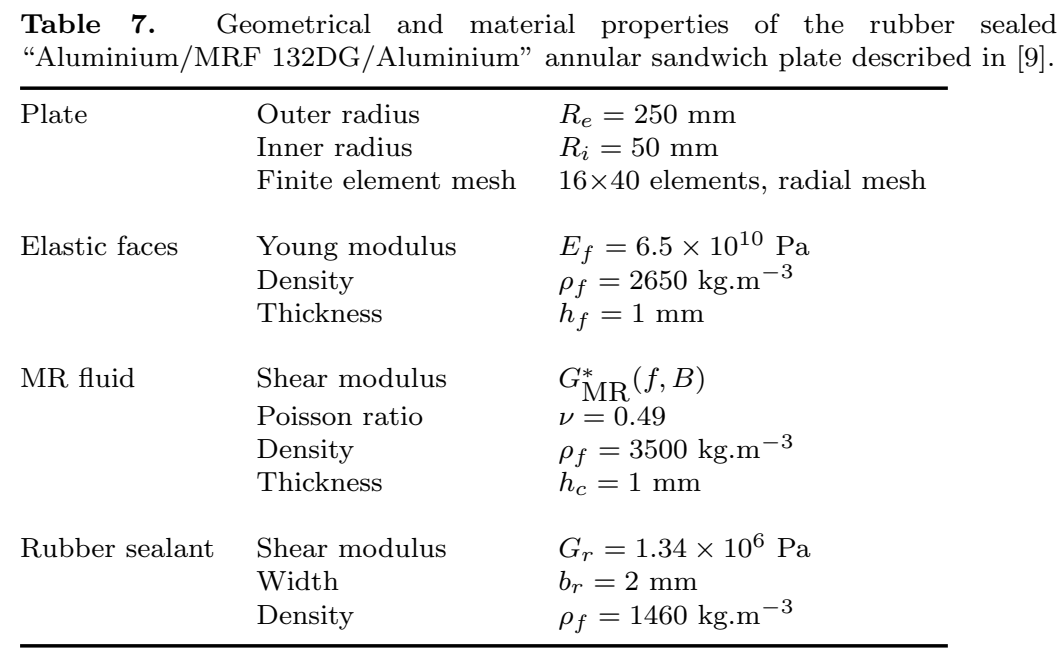

Table 8. Coefficients of the frequency and magnetic field dependent constitutive law 44-45 (from [9]).

\begin{tabular}{llll}
\hline$a_{0}$ & $192161 \mathrm{~Pa}$ & $b_{0}$ & $45524.4 \mathrm{~Pa}$ \\
$a_{1}$ & 30663.6 Pa.mT & $b_{1}$ & $812.4 \mathrm{~Pa}_{\mathrm{mT}}^{-1}$ \\
$a_{2}$ & $243.625 \mathrm{~Pa}^{-1} \mathrm{mT}^{-2}$ & $b_{2}$ & $6.4412{\mathrm{~Pa} . \mathrm{mT}^{-2}}^{-1}$ \\
$a_{3}$ & $0.00408 \mathrm{~Hz}^{-1}$ & $b_{3}$ & $0.00074 \mathrm{~Hz}^{-1}$ \\
\hline
\end{tabular}

In [9, the rubber and the MR fluid are homogenized as a single material. In the present FEM, we decide to implement them as two different materials.

On the one hand, the shear modulus of the MR fluid is modelled by a frequency and magnetic field dependent constitutive law [9]:

$$
G_{M R}^{*}(f, B)=G^{\prime}(f, B)+i G^{\prime \prime}(f, B),
$$

with

$$
G^{\prime}(f, B)=\left(a_{0}+a_{1} B+a_{2} B^{2}\right)\left(1-e^{-a_{3} f}\right),
$$

and

$$
G^{\prime \prime}(f, B)=\left(b_{0}+b_{1} B+b_{2} B^{2}\right)\left(1-e^{-b_{3} f}\right),
$$

where the frequency $f$ is in $\mathrm{Hz}$ and the magnetic field intensity $B$ varies in [0 mT, $90 \mathrm{mT}]$. The coefficients $a_{i}$ and $b_{i}$ are provided in table 8 .

On the other hand, the rubber seal is modelled by a viscoelastic material, the shear modulus of which is described by [9]:

$$
G_{r}(f)=1340000+i\left(152511+68.31 f+0.475 f^{2}\right),
$$

where $f$ is the frequency in Hz. Note that, the variations of the shear modulus regarding the frequency are negligible in the range of frequencies from $0 \mathrm{~Hz}$ to $100 \mathrm{~Hz}$, thus $G_{r}$ can be approximated by the constant law:

$$
G_{r}=1340000+i 152511 \text {. }
$$


To apply the proposed homotopy method, the frequency and magnetic field dependent constitutive law 43 is split into:

$$
G_{M R}^{*}(f, B)=G_{0}^{*}+G_{1}^{*}(f, B),
$$

where $G_{0}^{*}=G_{M R}^{*}(0,0)=0$ and $G_{1}^{*}(f, B)=G_{M R}^{*}(f, B)$. Referring to section 3, 48, , we propose the scaling:

$$
G_{1}^{*}(f, B)=\alpha G_{2}^{*}(f, B),
$$

where the parameter $\alpha$ of the second homotopy is chosen as:

$$
\alpha=F(B)=\frac{a_{0}+a_{1} B+a_{2} B^{2}}{a_{0}+a_{1} B_{\max }+a_{2} B_{\max }^{2}} .
$$

The second homotopy method is then run considering:

$$
G_{2}^{*}(f, B)=\kappa\left[\left(1-e^{-a_{3} f}\right)+i g(B)\left(1-e^{-b_{3} f}\right)\right],
$$

where

$$
\kappa=a_{0}+a_{1} B_{\max }+a_{2} B_{\max }^{2}
$$

and

$$
g(B)=\frac{b_{0}+b_{1} B+b_{2} B^{2}}{a_{0}+a_{1} B+a_{2} B^{2}} .
$$

Under these assumptions, (7) is a rational eigenvalue problem [30, 38.

Computed frequencies and loss factors are reported in table 9 . Results obtained by the double homotopy method (continuous variation of $B$ ) are in a very good agreement with those obtained by the incremental method (prescribed values for $B, 9]$ ). The differences in the loss factors result from the differences in the finite element modelling of the heterogeneous core properties.

Continuous branches of solutions presented in figure 7 reflect the nonlinearity of the constitutive law with respect to the magnetic field intensity. In particular, a decay of the loss factor of the first mode is observed for larger $B$. One notices that the incremental method does not exhibit this decay because too few points were reported in 9. A dichotomy method applied to the Taylor series of the loss factor of the first mode then indicates that the maximal damping occurs near to $B \simeq 73.43 \mathrm{mT}$.

\section{Conclusion}

The vibration analysis of smart rheological sandwich structures is carried out through a combination of numerical methods 22. The finite element method is applied to the free vibration analysis in order to write the complex nonlinear eigenvalue problem in a matrix form. The homotopy method allows for the solution of such a complex eigenvalue problem. In addition, the continuous nature of any homotopy can be accounted through Taylor series approximations and automatic differentiation that provides generality to the proposed nonlinear continuous eigensolver. These methods allow for the implementation of a numerical eigenvalue solver that notably allows for to the analysis of the continuous variation of the resonant frequencies and the loss factors with respect to the continuous variation of one of the modelling parameters.

The proposed method is exemplified on three different ER and MR sandwich structures, considering the continuous variations of ER/MR laws proposed in the literature with respect to their field intensity parameter and/or the frequency. 
Continuous eigenvalue solver for the design of ER/MR sandwich structures

Table 9. Rubber sealed "Aluminium/MRF 132DG/Aluminium" annular plate. Comparison of the first three resonance frequencies, loss factors and relative errors.

\begin{tabular}{|c|c|c|c|c|c|c|}
\hline \multirow[t]{2}{*}{$B(\mathrm{mT})$} & \multicolumn{2}{|c|}{$\begin{array}{l}\text { Homotopy } \\
\text { method }\end{array}$} & \multicolumn{2}{|c|}{$\begin{array}{l}\text { Incremental } \\
\text { method [9] }\end{array}$} & \multicolumn{2}{|c|}{ Relative error } \\
\hline & $f(\mathrm{~Hz})$ & $\eta$ & $f(\mathrm{~Hz})$ & $\eta$ & $f(\%)$ & $\eta(\%)$ \\
\hline \multirow[t]{3}{*}{0} & 15.93 & 0.039 & 15.67 & 0.040 & 1.66 & 2.50 \\
\hline & 43.14 & 0.021 & 41.96 & 0.017 & 2.81 & 23.53 \\
\hline & 78.52 & 0.018 & 71.21 & 0.018 & 10.27 & 0.00 \\
\hline \multirow[t]{3}{*}{30} & 18.74 & 0.124 & 18.67 & 0.123 & 0.37 & 0.81 \\
\hline & 48.07 & 0.076 & 47.20 & 0.077 & 1.84 & 1.30 \\
\hline & 87.91 & 0.073 & 85.79 & 0.071 & 2.47 & 2.82 \\
\hline \multirow[t]{3}{*}{50} & 21.25 & 0.167 & 22.06 & 0.160 & 3.67 & 4.38 \\
\hline & 52.38 & 0.109 & 52.09 & 0.105 & 0.56 & 3.81 \\
\hline & 96.17 & 0.105 & 94.62 & 0.096 & 1.64 & 9.38 \\
\hline \multirow[t]{3}{*}{90} & 27.13 & 0.182 & 28.87 & 0.174 & 6.03 & 4.60 \\
\hline & 62.94 & 0.143 & 62.77 & 0.127 & 0.27 & 12.60 \\
\hline & 116.31 & 0.135 & 115.29 & 0.119 & 0.88 & 13.45 \\
\hline
\end{tabular}

Resonance frequencies

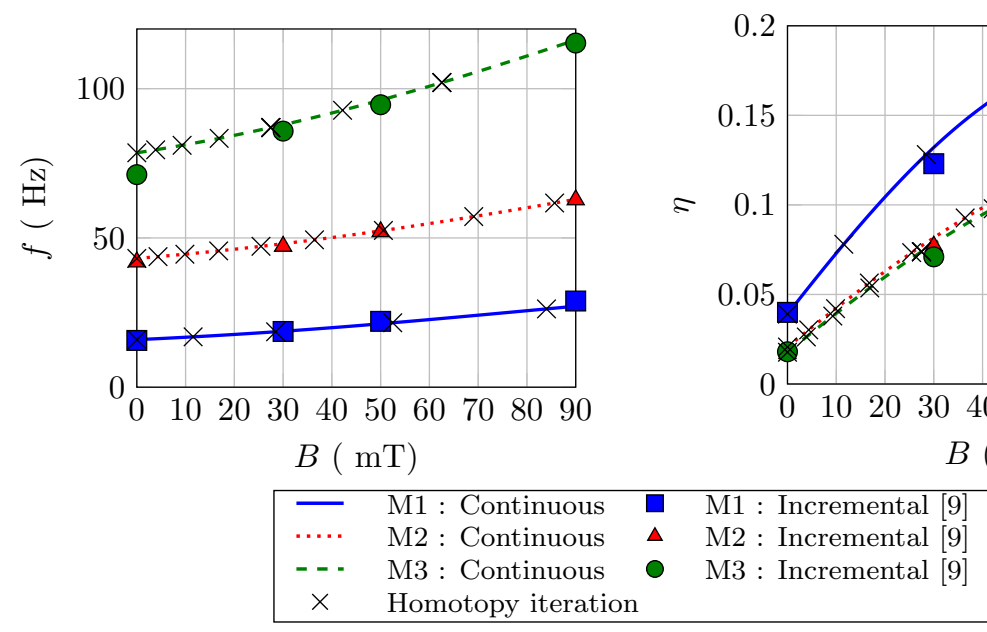

Loss factors

Figure 7. Rubber sealed "Aluminium/MRF 132DG/Aluminium" annular plate. Comparison of the first three resonance frequencies and loss factors computed by means of the homotopy method (continuous solid/dashed or dotted lines) and the incremental methods (coloured markers).

Calculation are carried out with respect to actual continuous variations of the intensity parameters. The continuous variations we present are in very good agreement with the related published discrete results. Where possible, we extended the computation of the resonant frequencies and loss factors over the whole range of validity of the constitutive law.

For a given mode, this continuous method allows for the exploration of the effects of the field intensity on the structural behaviour over a broad range. It notably highlights possible decays for larger field intensity variations, that were not exhibited in [9, 36] that use the incremental approach and a very few points to represent the loss factor variations. The maximal damping can then be determined by applying the dichotomy method to its Taylor series with little additional computational efforts. 
The proposed homotopy based method is thus of great interest for the design and the vibration control of smart materials and structures. The same methodology can be applied to the analysis of sandwich structures with respect to temperature variations.

\section{References}

[1] Nashif A D, Jones D I G and Henderson J P 1985 Vibration damping (John Wiley \& Sons) ISBN 978-0-471-86772-2

[2] Yalcintas M and Dai H 2004 Smart Mater. Struct. 13 1-11 ISSN 0964-1726, 1361-665X

[3] Zhou X Q, Yu D Y, Shao X Y, Zhang S Q and Wang S 2016 Compos. Struct. 136 460-480 ISSN 0263-8223

[4] Carlson J D, Coulter J P and Duclos T G 1990 Electrorheological fluid composite structures

[5] Weiss K D, Duclos T G, Chrzan M J and Yanyo L C 1996 Magnetorheological fluid composite structures

[6] Vishnu Narayana G and Ganesan N 2007 Compos. Struct. 80 221-233 ISSN 02638223

[7] Nayak B, Dwivedy S and Murthy K 2011 J. Sound Vib. 330 1837-1859 ISSN 0022460X

[8] De Souza Eloy F, Gomes G F, Ancelotti A C, da Cunha S S, Bombard A J F and Junqueira D M 2018 Engineering Structures 176 231-242 ISSN 0141-0296

[9] Eshaghi M, Sedaghati R and Rakheja S 2016 J. Intell. Mater. Syst. Struct. 27 2003-2037 ISSN 1045-389X, 1530-8138

[10] Ying Z and Ni Y 2017 Theoretical and Applied Mechanics Letters 7 61-66 ISSN 20950349

[11] De Souza Eloy F, Gomes G F, Ancelotti A C, da Cunha S S, Bombard A J F and Junqueira D M 2019 Composite Structures 209 242-257 ISSN 0263-8223

[12] Mohammadi N, Mahjoob M J, Kaffashi B and Malakooti S 2010 J. Mech. Sci. Technol. 24 1829-1837 ISSN 1738-494X

[13] Eshaghi M, Rakheja S and Sedaghati R 2015 Smart Mater. Struct. 24065018 ISSN 0964-1726

[14] Naji J, Zabihollah A and Behzad M 2016 Mech. Res. Commun. 77 50-59 ISSN 00936413

[15] Mohammadi F and Sedaghati R 2012 J. Intell. Mater. Syst. Struct. 23 1093-1105 ISSN 1045-389X

[16] Mohammadi F and Sedaghati R 2012 J. Intell. Mater. Syst. Struct. 23 1679-1697 ISSN 1045-389X

[17] Mohammadi F and Sedaghati R 2012 Smart Mater. Struct. 21075035 ISSN 0964-1726

[18] Rajamohan V, Sedaghati R and Rakheja S 2010 Smart Mater. Struct. 19015013 ISSN 0964-1726, 1361-665X

[19] Manoharan R, Vasudevan R and Jeevanantham A K 2014 Smart Mater. Struct. 23 025022 ISSN 0964-1726, 1361-665X 
[20] Eshaghi M, Sedaghati R and Rakheja S 2016 Compos. Struct. 157 78-86 ISSN 02638223

[21] Eshaghi M, Sedaghati R and Rakheja S 2017 Mech. Syst. Signal Process. 82 80-102 ISSN 0888-3270

[22] Bilasse M, Charpentier I, Daya E M and Koutsawa Y 2009 Comput. Methods. Appl. Mech. Eng. 198 3999-4004 ISSN 00457825

[23] He J H 2003 Appl. Math. Comput. 135 73-79 ISSN 0096-3003

[24] Charpentier I and Lampoh K 2016 Appl. Math. Model. 40 3365-3380 ISSN 0307904X

[25] Koutsawa Y, Charpentier I, Daya E M and Cherkaoui M 2008 Comput. Methods Appl. Mech. Eng. 198 572-577 ISSN 00457825

[26] Lampoh K, Charpentier I and Daya E M 2011 Comput. Methods Appl. Mech. Eng. 200 2983-2990 ISSN 00457825

[27] Lampoh K, Charpentier I and El Mostafa D 2014 CR Mécanique 342 700-705 ISSN 16310721

[28] Akoussan K, Boudaoud H, Daya E M, Koutsawa Y and Carrera E 2016 Compos. Struct. 149 11-25 ISSN 02638223

[29] Akoussan K, Boudaoud H, Daya E M, Koutsawa Y and Carrera E 2017 Mech. Adv. Mater. Struc. 1-13 ISSN 1537-6494, 1537-6532

[30] Betcke T, Higham N J, Mehrmann V, Schröder C and Tisseur F 2013 ACM Trans. Math. Softw. 39 7:1-7:28 ISSN 0098-3500

[31] Daya E M and Potier-Ferry M 2001 Comput. Struct. 79 533-541 ISSN 0045-7949

[32] Charpentier I 2012 Optim. Methods Softw. 27 221-232 ISSN 1055-6788, 1029-4937

[33] Yeh J Y and Chen L W 2004 Compos. Struct. 65 251-258 ISSN 02638223

[34] Bilasse M, Azrar L and Daya E 2011 Computers \& Structures 89 539-555 ISSN 00457949

[35] Don D L 1993 Theses and Dissertations 133

[36] Rajamohan V, Sundararaman V and Govindarajan B 2013 Procedia Eng. 64 603-612 ISSN 18777058

[37] Bilasse M, Daya E and Azrar L 2010 Journal of Sound and Vibration 329 49504969 ISSN 0022460X

[38] Kublanovskaya V N 1999 Journal of Mathematical Sciences 96 3085-3287 ISSN 1573-8795 